\title{
Pressure wave propagation in Managed Pressure Drilling- model comparison with real life data
}

\author{
Christian Berg ${ }^{1,3} \quad$ Jon Åge Stakvik ${ }^{2,3} \quad$ Bernt Lie $^{1} \quad$ Knut Vaagsaether $^{1}$ Glenn-Ole Kaasa ${ }^{3}$ \\ ${ }^{1}$ Department of Electrical Engineering, IT and Cybernetics, University of South-Eastern Norway, Norway, \\ Bernt.Lie@usn.no \\ ${ }^{2}$ Department of Engineering Cybernetics, Norwegian University of Science and Technology, Norway \\ ${ }^{3}$ Kelda Drilling Controls, Norway, cbe@kelda.no
}

\begin{abstract}
Drilling for oil and gas is a complex process, involving pumping of fluid through kilometers of pipes. Even though the drilling fluid has a high speed of sound $(\approx 1000$ $\mathrm{m} / \mathrm{s}$ ), the large lengths involved make pressure wave propagation significant in timescales where such phenomena can usually be neglected in other processes. Managed pressure drilling, a technological extension of conventional drilling, adds a choke on the return flow from the drilling process. Significant work has been done in recent years on creating a simplified model of the process, often by neglecting distributed dynamics, and using this for controller design. This paper compares the simplified model most often used, with a distributed partial differential equation (PDE) model and compare the performance with measured data for wave propagation while doing managed pressure drilling. Fluid structure interaction and theoretical vs recorded speed of sound are discussed.
\end{abstract}

Keywords: managed pressure drilling, PDE, wave propagation, FSI

\section{Introduction}

Managed pressure drilling (MPD), today considered an "unconventional" drilling technology, is a natural technological advancement of conventional drilling. MPD is forecasted to grow significantly in the future, with key industry players indicating that it might be the new "conventional" in the near future. Drilling for oil and gas is a complex process with complex dynamic behaviour. The dynamics of the entire system has to be understood for controller and estimator design if the prognosed future growth and adoption is to be achieved.

For MPD, significant work has been done in recent years by control engineers/researchers on simplifying the mathematical model for the process to aid in controller and estimator design. A schematic view of the process is given in Figure 1. The most often used of these simplified models is the one by (Kaasa et al., 2012). Multiple estimation and control strategies based on this simplified model has been published (Stakvik et al., 2016; Stakvik et al., 2017; Zhou et al., 2011; Stamnes et al., 2008;

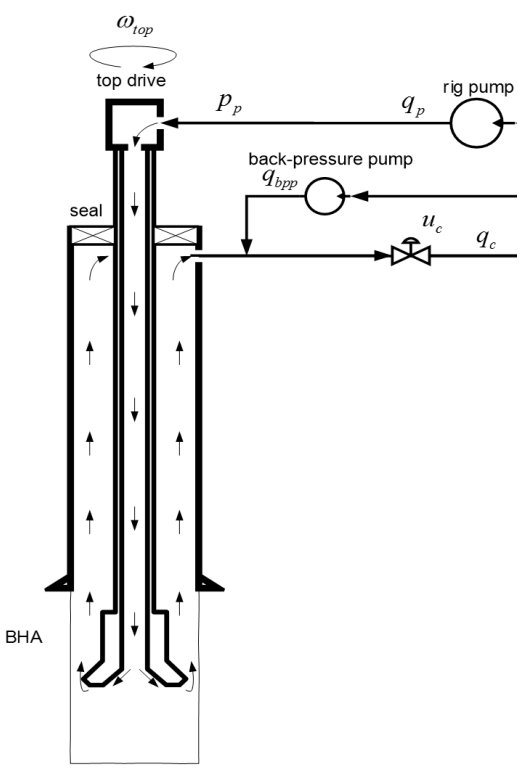

Figure 1. Managed pressure drilling. Drilling fluid is circulated from the rig mud pumps and down the drill string. At the bottom of the well bore the drilling fluid flows out through the drill bit via nozzles, and is then circulated up to the surface in the annular space between the drill string and annulus.

Hauge et al., 2012). There is also ongoing research on designing estimators and controllers based on a linearised PDE distributed model (Aarsnes et al., 2014; Aarsnes et al., 2012; Anfinsen and Aamo, 2018). To verify design, controllers and estimators should in general always be tested on a system model that is higher fidelity than the model the design is based on to ensure that something critically important was not forgotten in the simplification. This paper compares the response of the simplified model by (Kaasa et al., 2012) with a PDE based model for the process, and compare this to real drilling data from MPD operations.

\section{Model}

Considering the process shown in Figure 1 and conservation of mass and momentum, dynamic models for the process can be derived. The model by (Kaasa et al., 2012) 
disregards distributed effects in the drill string and annulus, and consider these as two volumes where mass should be conserved. To capture wave propagation, distributed effects should be considered, making the drillstring and annulus take the form of partial differential equations (Di Meglio and Aarsnes, 2015).

\subsection{Simplified model}

The ODE model presented in (Kaasa et al., 2012) and given in (1-5) can be derived with the following assumptions: The drill string and annulus are treated as two volumes where mass is conserved, the drill string pressure loss as quadratic with flow (turbulent), the drill bit pressure loss as quadratic with flow, and the annulus pressure loss as linear with flow (laminar). Note that here, the annulus and drill string volumes are considered constant, and in and out flow of the drilled formation is not considered.

$$
\begin{gathered}
\frac{V_{d}}{\beta_{d}} \frac{\mathrm{d} p_{p}}{\mathrm{~d} t}=q_{p}-q_{b i t} \\
\frac{V_{a}}{\beta_{a}} \frac{\mathrm{d} p_{c}}{\mathrm{~d} t}=q_{b i t}+q_{b p p}-q_{c} \\
M \frac{\mathrm{d} q_{b i t}}{\mathrm{~d} t}=p_{p}-p_{c}-F_{d} q_{b i t}^{2}-F_{a} q_{b i t} \\
M=\int_{0}^{L_{d}} \frac{\rho_{d}}{A_{d}(x)} \mathrm{dx}+\int_{L_{a}}^{0} \frac{\rho_{a}}{A_{a}(x)} \mathrm{dx} \\
q_{c}=g_{c}\left(z_{c}\right) K_{c} \sqrt{\frac{2}{\rho}\left(p_{c}-p_{c o}\right)}
\end{gathered}
$$

In (1-5) $V_{d}$ and $V_{a}$ are drill string and annulus volumes, $\beta_{d}$ and $\beta_{a}$ are fluid modulus of compressibility $\beta=\frac{1}{\rho} \frac{\mathrm{d} \rho}{\mathrm{d} p}, q_{p}$ is the pump flow, $q_{b i t}$ is a state representing flow from the drill string to the annulus, $F_{d}$ and $F_{a}$ are friction factors for the drill string and annulus, $g_{c}$ is choke area as a function of choke position $z_{c}, \rho_{a}, \rho_{d}$ are fluid densities in drill string and annulus, and $A_{d}, A_{a}$ are flow cross sectional areas.

\subsection{PDE model}

If distributed effects are considered, the drill string and annulus can be modelled using the PDE system given in (6-7) representing conservation of mass and momentum, respectively.

$$
\begin{gathered}
\frac{\partial \rho}{\partial t}+\frac{\partial \rho u}{\partial x}=0 \\
\frac{\partial \rho u}{\partial t}+\frac{\partial\left(\rho u^{2}+p\right)}{\partial x}=-f(\rho, u)-g(\rho)
\end{gathered}
$$

where $\rho$ is density and $u$ is velocity.

Putting (6-7) in vector form as in (8) and introducing temporary variables $u_{1}, u_{2}$.

$$
\frac{\partial \mathrm{U}}{\partial t}+\frac{\partial}{\partial x}(F(\mathrm{U}))=S(\mathrm{U})
$$

$$
\begin{array}{r}
\mathrm{U}=\left[\begin{array}{c}
\rho \\
\rho u
\end{array}\right]=\left[\begin{array}{l}
u_{1} \\
u_{2}
\end{array}\right] \\
F(\mathrm{U})=\left[\begin{array}{c}
\rho u \\
\rho u^{2}+p
\end{array}\right]=\left[\begin{array}{c}
u_{2} \\
\frac{u_{2}^{2}}{u_{1}}+\frac{\partial p}{\partial \rho} u_{1}
\end{array}\right] \\
S(\mathrm{U})=\left[\begin{array}{c}
0 \\
0 \\
-f(\rho, u)-G(\rho, \theta)
\end{array}\right]=\left[\begin{array}{c}
\left.0, \frac{u_{2}}{u_{1}}\right)-G\left(u_{1}, \theta\right)
\end{array}\right]
\end{array}
$$

Where $\partial p=\frac{\partial p}{\partial \rho} \partial \rho$ is used for removing $p$ in (9). Then in pseudo linear form as

$$
\begin{gathered}
\frac{\partial \mathrm{U}}{\partial t}+A(\mathrm{U}) \frac{\partial \mathrm{U}}{\partial x}=S(\mathrm{U}) \\
A(\mathrm{U})=\frac{\partial F(\mathrm{U})}{\partial \mathrm{U}}=\left[\begin{array}{cc}
0 & 1 \\
-\frac{u_{2}^{2}}{u_{1}^{2}}+\frac{\partial p}{\partial \rho} & 2 \frac{u_{2}}{u_{1}}
\end{array}\right]
\end{gathered}
$$

it can be found that the eigenvalues of $A(\mathrm{U})$ are $\lambda_{1,2}=u \pm \sqrt{\frac{\partial p}{\partial \rho}}$ where $\sqrt{\frac{\partial p}{\partial \rho}}$ is the speed of sound in the fluid.

The source terms $f(\rho, u)$ and $G(\rho, \theta)$ represent friction and hydrostatic pressure due to gravity, respectively. Friction is modeled as (12)

$$
\begin{array}{r}
f(\rho, u)=\frac{1}{2} K_{\text {fric }} f \rho u^{2} \\
f=\max \left(\frac{64}{\operatorname{Re}}, \frac{0.25}{\left(\log \left(\frac{\varepsilon}{3.7 D}+\frac{5.74}{\operatorname{Re}^{0.9}}\right)\right)^{2}}\right) \\
\operatorname{Re}=\frac{\rho u D}{\mu}
\end{array}
$$

where $f$ is the Darcy friction factor, Re is the Reynolds number, $\varepsilon$ is the surface roughness of the pipe, and $D$ is the hydraulic diameter. $f=\frac{64}{\mathrm{Re}}$ represents laminar flow, $f=\frac{0.25}{\left(\log \left(\frac{\varepsilon}{3.7 D}+\frac{5.74}{\mathrm{R}^{0} 0.9}\right)\right)^{2}}$ is an approximation (Swamee and K. Jain, 1976) to the Colebrook equation, and the maximum of these two is taken to cover both laminar and turbulent regimes. $K_{\text {fric }}$ is a tuning factor to fit measured field data, ideally set to 1 .

Hydrostatic pressure is modelled as (13) where $\theta$ is the local angle between the well bore and the horizontal.

$$
G(\rho, \theta)=\rho g \sin (\theta)
$$

There are numerous numerical approaches to solving the PDE system in (6-7), (Vytvytsky and Lie, 2017), (Palacios G and Da Silva, 2013) both with and without considering fluid structure interaction. The details of different methods for solving (6-7) with strengths and weaknesses is not elaborated in detail in this paper. Here a staggered grid approach is used.

For simulation, (6) is transformed into an equation for pressure. Assuming the density can be given as a linear function of pressure as in (14), (6-7) can be rewritten as (15-16). 


$$
\begin{gathered}
\rho=\rho_{0}+\frac{\rho_{0}}{\beta}\left(p-p_{0}\right) \\
\frac{d \rho}{d p}=\frac{\rho_{0}}{\beta} \\
\frac{\rho_{0}}{\beta} \frac{\partial p}{\partial t}+\frac{\partial \rho u}{\partial x}=0 \\
\frac{\partial \rho u}{\partial t}+\frac{\partial \rho u^{2}}{\partial x}=-\frac{\partial p}{\partial x}-f(\rho, u)-g(\rho)
\end{gathered}
$$

$$
\begin{gathered}
\rho u A_{a n}(x=L)=\rho u A_{d s}(x=L) \\
p_{a n}(x=0)=p_{c}
\end{gathered}
$$

(14) The system solved is given in (29-30) where the pressure equation is solved in the grid cell centre and the flow equation is solved on a grid that has the cell centre on the pressure grid face.

$$
\begin{gathered}
\frac{\partial p}{\partial t}=-\frac{\beta}{V \rho_{0}}\left((q \rho)_{\text {out }}-(q \rho)_{\text {in }}\right) \\
\frac{\partial q}{\partial t}=-q \frac{\rho_{0}}{\rho \beta} \frac{\partial p}{\partial t}-\frac{1}{\rho L}\left(\left(\frac{q^{2} \rho}{A}\right)_{\text {out }}-\left(\frac{q^{2} \rho}{A}\right)_{\text {in }}\right) \ldots \\
-\frac{A}{\rho L}\left(p_{\text {out }}-p_{\text {in }}\right)+S_{x} \frac{A}{\rho}
\end{gathered}
$$
steeg and Malalasekera, 2019).

$$
\begin{gathered}
\oint_{C V}\left(\frac{\rho_{0}}{\beta} \frac{\partial p}{\partial t}+\frac{\partial \rho u}{\partial x}\right) d V=0 \\
\oint_{C V}\left(\frac{\partial \rho u}{\partial t}+u \frac{\partial \rho u}{\partial x}\right) d V=\oint_{C V}\left(-\frac{\partial p}{\partial x}+S_{x}\right) d V
\end{gathered}
$$

Applying the Gauss divergence theorem; $\oint_{C V} \operatorname{div}(\phi u) d V=\oint_{S} \mathbf{n} \cdot(\phi u) d S$

$$
\begin{gathered}
\oint_{C V}\left(\frac{\rho_{0}}{\beta} \frac{\partial p}{\partial t}\right) d V+\int_{A} \mathbf{n} \cdot(\rho u) d A=0 \\
\oint_{C V}\left(\frac{\partial \rho u}{\partial t}\right) d V+\int_{A} \mathbf{n} \cdot\left(\rho u^{2}\right) d A=\oint_{C V}\left(-\frac{\partial p}{\partial x}+S_{x}\right) d V
\end{gathered}
$$

Solving the integrals and discretizing in space yield (21-22).

$$
\begin{gathered}
V \frac{\rho_{0}}{\beta} \frac{\partial p}{\partial t}+(u A \rho)_{o u t}-(u A \rho)_{\text {in }}=0 \\
V \frac{\partial \rho u}{\partial t}+\left(u^{2} A \rho\right)_{\text {out }}-\left(u^{2} A \rho\right)_{\text {in }}=-V \frac{p_{\text {out }}-p_{\text {in }}}{L}+S_{x} V
\end{gathered}
$$

Doing the variable change $q=u A$, applying the chain rule to $\frac{\partial \rho u}{\partial t}$, and using that $q \frac{\partial \rho}{\partial t}=q \frac{\rho_{0}}{\beta} \frac{\partial p}{\partial t}$ yield (23-24).

$$
\begin{gathered}
V \frac{\rho_{0}}{\beta} \frac{\partial p}{\partial t}+(q \rho)_{\text {out }}-(q \rho)_{\text {in }}=0 \\
\frac{V}{A}\left(\rho \frac{\partial q}{\partial t}+q \frac{\rho_{0}}{\beta} \frac{\partial p}{\partial t}\right)+\left(\frac{q^{2} \rho}{A}\right)_{\text {out }}-\left(\frac{q^{2} \rho}{A}\right)_{\text {in }} \cdots \\
\ldots=-A\left(p_{\text {out }}-p_{\text {in }}\right)+S_{x} V
\end{gathered}
$$

With boundary conditions

$$
\begin{gathered}
q_{d s}(x=0)=q_{p} \\
p_{d s}(x=L)=p_{a n}(x=L)+\frac{1}{K_{\text {nozzle }}}\left(\frac{q_{d s}(x=L)}{A_{\text {nozzle }}}\right)^{2}
\end{gathered}
$$

The spatial arrangement of states can be seen for an example case with $n=3$ grid elements for flow and $n+1$ grid elements for pressure in Figure 2. The subscript $g$ in $p_{0, g}$ and $p_{n+1, g}$ is to represent that this is a "ghost node". Ghost nodes are grid elements outside of the physical domain used to implement boundary conditions.

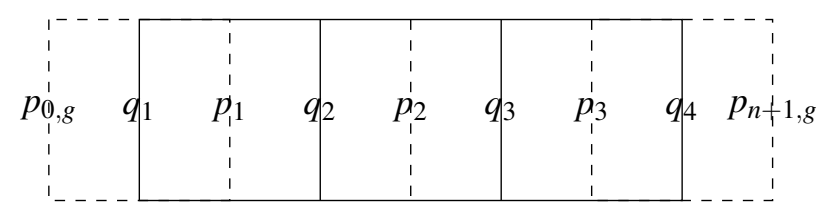

Figure 2. Staggered grid showing the spatial staggering of the system solved. If $i$ represent grid number on the flow grid for $q$, and $k$ represent grid number for the pressure grid for variables $p$ and $\rho$, note that $i+\frac{1}{2}=k, k+\frac{1}{2}=i+1$

From Figure 2 it can be seen that $q_{\text {out }}, q_{\text {in }}$ (being $q_{2}$ and $q_{1}$ respectively for $p_{1}$ ) and $p_{\text {out }}, p_{\text {in }}$, (being $p_{1}$ and $p_{0, g}$ for $q_{1}$ ) is known directly due to the spatial staggering of states.

Variables that are not directly available on grid faces from the staggered arrangement ( $\rho$ in (29); $q$ and $q \frac{\rho_{0}}{\beta} \frac{\partial p}{\partial t}$ in (30)) are found by using a first order up-winding in flow, as in (31)

$$
\theta_{i+\frac{1}{2}}= \begin{cases}\theta_{i} & q>0 \\ \theta_{i+1} & q<0 \\ \frac{\theta_{i}+\theta_{i+1}}{2} & q=0\end{cases}
$$

Equations (29-30) are solved in time by using a 4th order Runge Kutta method.

\subsubsection{A brief discussion on equation of state}

Using (14) as an Equation of State for the liquid will yield a speed of sound from the eigenvalue analysis in (10) as a function of $\rho_{0}$ and $\beta$ given in (32)

$$
c=\sqrt{\frac{\beta}{\rho_{0}}}
$$


Drilling fluids are in most cases a mixture of water and weighting material (water based mud, WBM), oil, water and weighting material (oil based mud, OBM) or synthetic oil, water, and weighting material (Synthetic based mud, SBM). Drilling fluids usually also contain a small fraction of additives (emulsifiers, gelling agents, etc.), at a low volume fraction. The equivalent mixture bulk modulus should be found for use in (14) (Carcione and Poletto, 2000). Although all fluid components are only slightly compressible, the weighting material can be treated as incompressible compared to the water and oil. The volume fraction of additives are neglected here.

The mixture bulk modulus $\beta_{m}$ can be found as in (33) where subscripts $w, o, s$ denote water, oil and solids respectively.

$$
\frac{1}{\beta_{m}}=\frac{\alpha_{w}}{\beta_{w}}+\frac{\alpha_{o}}{\beta_{o}}+\frac{\alpha_{s}}{\beta_{s}}
$$

Here $\alpha_{i}$ is the volume fraction of that mixture component. Note that $\alpha_{w}+\alpha_{o}+\alpha_{s}=1$. Assuming the solids component to be incompressible as $\beta_{s}>>\beta_{o}, \beta_{w}$, analogous to saying $\beta_{s}=\infty$ makes the last term on the RHS of (33) disappear.

The mixture density $\rho_{m 0}$ can be found as (34)

$$
\rho_{m 0}=\alpha_{w} \rho_{w 0}+\alpha_{o} \rho_{o 0}+\alpha_{s} \rho_{s 0}
$$

In practice, a pre-defined ratio of oil/water is used when mixing the drilling fluid, and then weighting solids is added to reach the desired liquid density. For WBM fluids there is no oil fraction, and solids are added to reach the desired density. This can be used to further simplify (33-34). By using oil-water ratio, $R_{o w}=\frac{\alpha_{o}}{\alpha_{w}}$, and the fact that the sum of all the component volume fractions is 1, (33-34) can be rewritten in forms that are simple for straight forward use, as given in (35-36) where the inputs are the mixture and component densities $\rho_{m}, \rho_{i}$, component compressibility $\beta_{i}$, and oil-water ratio $R_{o w}$.

$$
\begin{gathered}
\alpha_{s}=\frac{\rho_{m}-\rho_{w}+R_{o w}\left(\rho_{m}-\rho_{o}\right)}{\rho_{s}-\rho_{w}+R_{o w}\left(\rho_{s}-\rho_{o}\right)} \\
\beta_{m}=\frac{\beta_{w} \beta_{o}\left(1+R_{o w}\right)}{\beta_{o}\left(1-\alpha_{s}\right)+R_{o w} \beta_{w}\left(1-\alpha_{s}\right)}
\end{gathered}
$$

For water based mud, $R_{o w}=0$ and (35-36) are still valid. Equations (35-36) are only valid at a given pressure as the volume fractions change with pressure. In practice the effect of this is minor.

\subsubsection{Fluid structure interactions (FSI)}

If fluid structure interactions are considered, i.e., the flow cross sectional area changes with pressure, an equivalent bulk modulus $\beta_{e}$ can be calculated and used in $(23,24)$. Note that the mixture bulk modulus $\beta_{m}$ should still be used in the liquid Equation of State in (14).

Taking pipe expansion into account, equivalent bulk modulus can be calculated as (37). Here the possible compression of the drill string inside the annulus is neglected.
For the full derivation of (37) in the context of the applied PDE, the reader is referred to (Carlsson, 2016).

$$
\beta_{e}=\frac{\beta_{m}}{\left(1+\frac{\beta_{m} D}{d E} \phi\right)}
$$

In (37), $\beta_{m}$ is mixture bulk modulus from (36), $E$ is Young's modulus of the pipe, $D$ is the pipe diameter, $d$ is the pipe wall thickness, and $\phi$ is the pipe support factor. Here axial stresses are neglected, setting $\phi=1$.

\subsubsection{Gridding}

In a real well geometry there are numerous changes in cross sectional area with axial position, mainly caused by the drill string consisting of different pipe sections screwed together. Spatial discretization (gridding) at the resolution required to capture all the changes exactly will require a large number of grid elements. Here, a gridding routine that ensures the grid volume and volume of the real geometry are exactly equal, is used. The real vs discretized geometry for the test well studied near the bottom hole assembly (BHA) is shown in Figure 3.

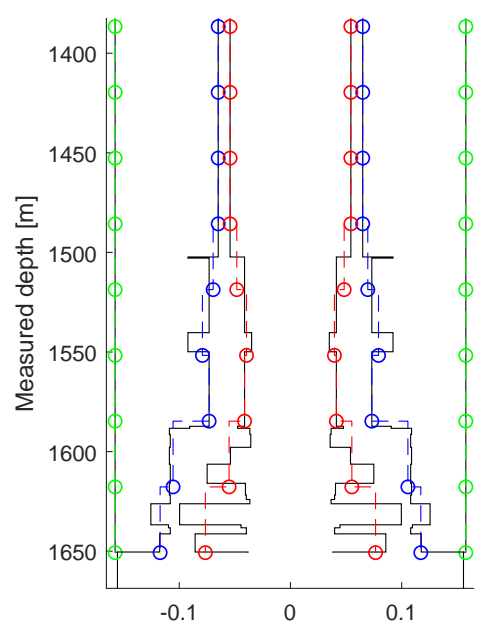

Figure 3. Volume conserving grid of bottom hole assembly (BHA). Solid black: Original geometry. Dashed green: Gridded well bore diameter (annulus). Dashed blue: Gridded drill string outer diameter (annulus). Dashed red: Gridded drill string inner diameter (drill string).

\subsubsection{Boundary conditions}

For comparison with field data, the algebraic relation between choke flow and choke pressure for the simplified model is skipped, and measured choke pressure is used directly as a boundary condition. This yields a simplified model with two ODE's, (as opposed to the three ODE's in the original model by (Kaasa et al., 2012)) specified in $(1,3,4)$. To compare the model's dynamic response to the measured data, the boundary values that are not specified are compared to measured data. That is, measured pump flow and choke pressure are used as boundary conditions. 
Then, simulated and measured pump pressure and choke flow are compared. The simplified model has no choke flow when the choke pressure is specified, so only pump pressure is compared to measured pump pressure.

For the PDE model the boundary conditions for $n$ grid elements are set as follows.

- Inlet

- $p_{0}=p_{1}$. That is, the inlet ghost node for pressure is set to the same value as the next grid element

- $q_{1}=q_{b c}$, the flow into the domain is specified

- Outlet

- $p_{n+1}$, the outlet ghost node for pressure is set to $2 p_{b c}-p_{n}$ where $p_{b c}$ is the specified boundary pressure

\subsection{Initial conditions}

For the PDE model the initial conditions is set to the hydrostatic pressure for $p$, that is $p_{i}=\rho_{0} g h_{i}$ where $h_{i}$ is the grid vertical depth. The initial condition for flow $q$ is set to zero. Flow is then ramped up to the flow rate in the start of case considered and simulation run for 150 seconds to reach steady state.

\section{Comparison with field data}

In MPD operations, if the choke controller is active, wave propagation phenomena are rarely visible. This is due to the choke pressure controller being used in the data the author has available is specifically designed to keep within the limits of the simplified model. During system commissioning, direct choke position control is used to verify calibration of the controller model, and pressure wave dynamics gets excited. When doing choke position control, the rate of change of the position is limited in the controller to about $\approx 5 \% / \mathrm{s}$ to avoid severe water hammer effects caused by the operator, but still fast enough that wave dynamics is excited. The controller in closed loop has access to the full choke actuator performance $\approx 25 \% / \mathrm{s}$, making the testing of the mentioned controller on a PDE model very important as it is easily able to excite wave dynamics in cases with improper tuning. For validation of the models with data, a time period from commissioning on a $1647 \mathrm{~m}$ deep offshore well is used, as seen in Figure 4. The commissioning is performed in "cased hole", that is, the annulus has a steel casing going all the way to the bottom of the well and there is no "open hole" (exposed reservoir) .

In Figure 4, the pump flow rate is near constant, and the choke is closed and then opened again 3 times at various speeds, giving an increase in choke pressure (boundary condition), and then an increase in pump pressure (modelled), governed by the pressure dynamics of the well. The choke flow changes when the choke position is changed.
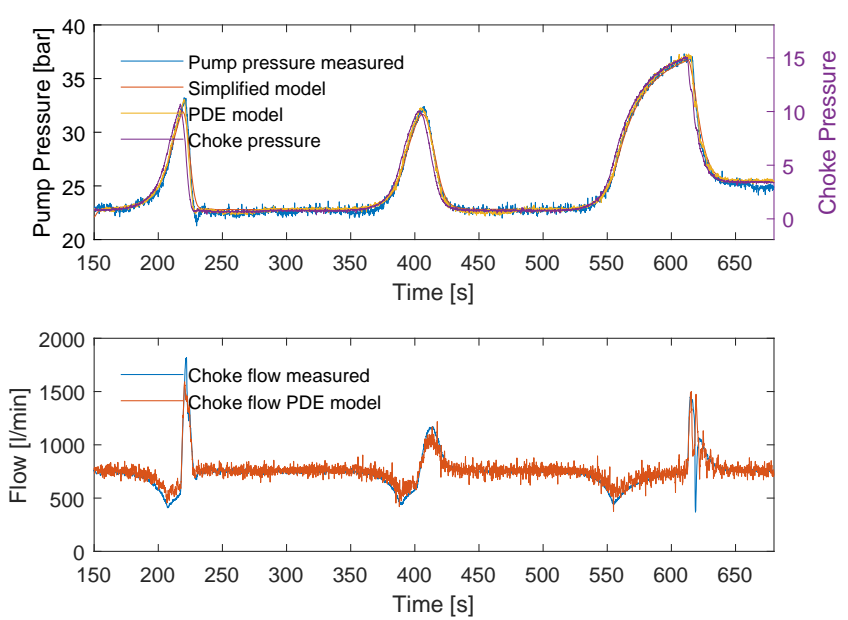

Figure 4. Steps in choke position during cased hole commissioning for $1647 \mathrm{~m}$ deep offshore well. Top: Pressure simulated vs measured. Top right axis: Measured choke pressure (boundary condition). Bottom: Simulated vs measured choke flow.

This is caused by the compression/expansion of liquid and possible expansion of well geometry due to pressure.

\subsection{Field data comparison, no fluid structure interactions}

Here the response to the choke position steps are studied for all steps individually. Fluid structure interactions are not considered. Parameters used in the PDE and simplified model are given in Table 1. A "fudge factor" $K_{\text {fric }}$ for friction in the PDE model was required to make the simulated pump pressure fit with the measured data. At the flow rates in the cases studied, the frictional pressure loss will be laminar in both the drill string and annulus. The assumption of Newtonian fluid in (12) is not really true for drilling fluids as they exhibit gelling behavior, something that will lead to a higher friction loss than for Newtonian fluids at low flow rates.

The noise on the pump pressure and choke flow in the PDE model is caused by noise on the choke pressure boundary condition. Filtering the noise on the signal is avoided as the phenomena studied are fast compared to the sampling rate. Figure 5 shows a close-up of the first step from Figure 4.

It is seen from Figure 5 that, qualitatively, the results of the PDE model fits reasonably well with the measured data. The PDE model under-predicts the changes of choke flow due to choke pressure. The response on pump pressure happens faster in the PDE model than in the measured data. This indicates that the wave propagation time in the PDE model is faster than in reality. The simplified model is able to predict pump pressure well when the pressure is increasing, but ends up giving a "smoothed" response on the more rapid opening of the choke.

Figure 6 shows the response in the second step, where both the increase and decrease of choke pressure is slower than that in the first step. It is clearly seen that as changes happen more slowly, the difference between the simplified 
Table 1. Model parameters.

\begin{tabular}{l|l|r} 
Parameter & Value & Unit \\
\hline$V_{d}$ & 15.27 & {$\left[\mathrm{~m}^{3}\right]$} \\
$V_{a}$ & 104.94 & {$\left[\mathrm{~m}^{3}\right]$} \\
$R_{o w}$ & 4 & {$[-]$} \\
$\rho_{m 0}=\rho_{d}=\rho_{a}$ & 1210 & {$\left[\mathrm{~kg} / \mathrm{m}^{3}\right]$} \\
$\rho_{w 0}$ & 1000 & {$\left[\mathrm{~kg} / \mathrm{m}^{3}\right]$} \\
$\rho_{o 0}$ & 850 & {$\left[\mathrm{~kg} / \mathrm{m}^{3}\right]$} \\
$\rho_{s 0}$ & 4200 & {$\left[\mathrm{~kg} / \mathrm{m}^{3}\right]$} \\
$\beta_{w}$ & $2.2 \mathrm{e} 9$ & {$[\mathrm{~Pa}]$} \\
$\beta_{o}$ & $1.5 \mathrm{e} 9$ & {$[\mathrm{~Pa}]$} \\
$\beta_{a}$ (eq.36) & $1.78 \mathrm{e} 9$ & {$[\mathrm{~Pa}]$} \\
$\beta_{d}($ eq.36) & $1.78 \mathrm{e} 9$ & {$[\mathrm{~Pa}]$} \\
$L_{d}$ & 1651 & {$[\mathrm{~m}]$} \\
$L_{a}$ & 1651 & {$[\mathrm{~m}]$} \\
$A_{d}$ & 0.0092 & {$\left[\mathrm{~m}^{2}\right]$} \\
$A_{a}$ & 0.0636 & {$\left[\mathrm{~m}^{2}\right]$} \\
$M$ (eq.4) & $2.47 \mathrm{e} 8$ & {$\left[\mathrm{~kg} / \mathrm{m}^{4}\right]$} \\
$\mu$ & $45 \mathrm{e}-3$ & {$[\mathrm{~Pa} \cdot \mathrm{s}]$} \\
$\varepsilon$ & $4.5 \mathrm{e}-5$ & {$[\mathrm{~m}]$} \\
$K_{\text {fric }}$ & 2.4 & {$[-]$} \\
$A_{\text {nozzle }}$ & $6.25 \mathrm{e}-04$ & {$\left[\mathrm{~m}^{2}\right]$} \\
$K_{\text {nozzle }}$ & 0.8 & {$[-]$}
\end{tabular}

model, PDE model, and measured data becomes smaller. This is reasonable as that the main difference between the simplified and PDE model is whether distributed pressure effects are neglected. The effect of choke pressure on choke flow in the PDE model is still under-predicted, as in the first pressure step.

Figure 7 shows the response of the simplified and PDE model compared to field data for the third pressure step. In this step, the opening of the choke is even faster than that of the case in Figure 5. Note the "wave" in measured choke pressure. As for the two first cases, the results of the PDE and simplified model compared to field data is very similar at the increase of pressure with different response on opening the choke quickly. The previous observation of choke flow being under-predicted in the PDE model is visible when the pressure is increased, but not that clearly visible when the choke is opened.

\subsection{Field data comparison, fluid structure in- teractions}

Here the steps in the previous section is revisited, with fluid structure interactions (FSI) considered. Parameters used when FSI is considered are given in Table 2.

Figure 8 shows the PDE model with and without FSI in the first step. Considering FSI through (37), yields a lower $\beta$ for the drill string and annulus, something that will increase the wave propagation time (decrease velocity) in the PDE model, as well as make the effect of choke pressure on choke flow be more significant. The assumption of no axial stresses used is not strictly true. For the annulus, the casing will mainly be under compression loads axially.
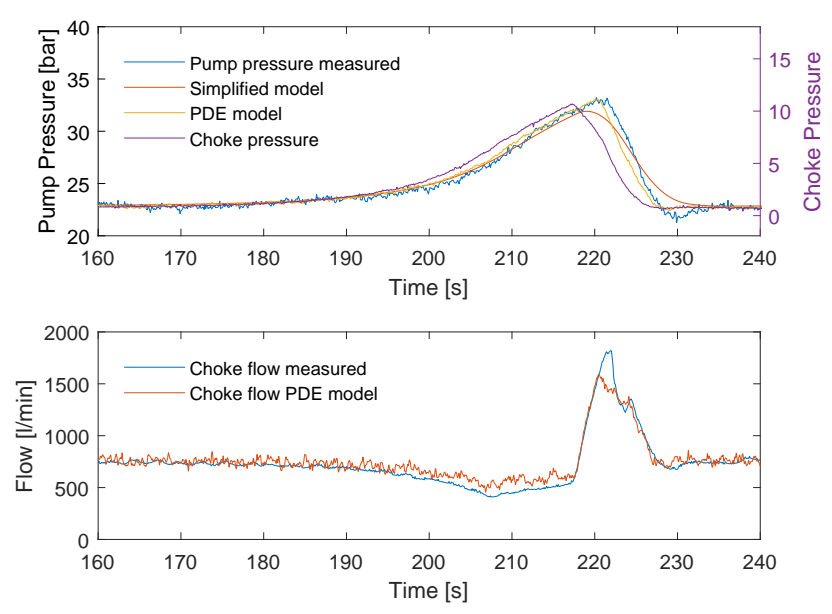

Figure 5. First step in choke position; slow closing and rapid opening of choke. Top: Pressure simulated vs measured. Top right axis: Measured choke pressure (boundary condition). Bottom: Simulated vs measured choke flow.
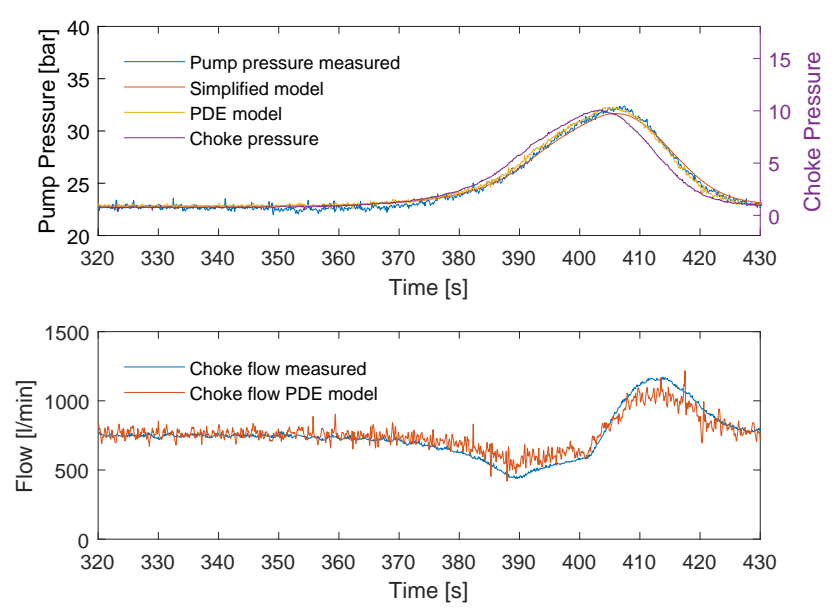

Figure 6. Second step in choke position; slow closing and opening of choke. Top: Pressure simulated vs measured. Top right axis: Measured choke pressure (boundary condition). Bottom: Simulated vs measured choke flow.

The neglected effect of compression of the drill string in the annulus together with the axial forces in the casing would likely lead to slightly lower effective bulk modulus. The drill string experiences both stretch and compression along the length.

As seen in Figure 8, the effect of choke pressure on flow becomes more significant when considering FSI, making the PDE model fit the measured flow data better compared to the model neglecting FSI. Wave propagation time reduces slightly when considering FSI, but there is still a mismatch between the PDE model and recorded data.

Figure 9 shows the PDE model compared to measured data for the second step in pressure, with and without FSI. Overall the results for the second pressure step are similar to the no FSI case, with the transient being slow enough that wave propagation effects are minor. The effect of choke pressure on choke flow compared to measured data is better when considering FSI than not considering FSI, 

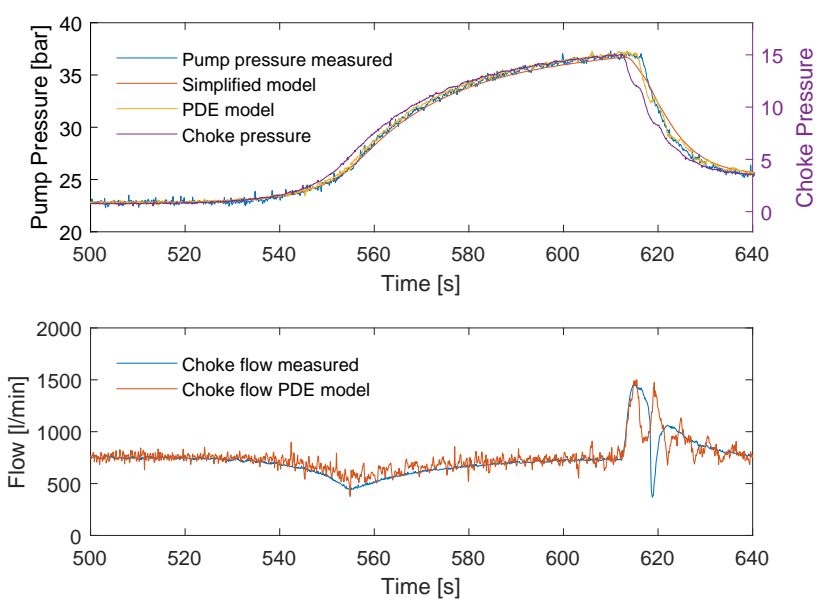

Figure 7. Third step in choke position; slow closing and very rapid opening of choke. Top: Pressure simulated vs measured. Top right axis: Measured choke pressure (boundary condition). Bottom: Simulated vs measured choke flow.

Table 2. Model parameters with FSI considered.

\begin{tabular}{l|l|l} 
Parameter & Value & $\mathrm{Unit}$ \\
\hline$\beta_{m}$ (eq.36) & $1.78 \mathrm{e} 9$ & {$[\mathrm{~Pa}]$} \\
$E$ & $200 \mathrm{e} 9$ & {$[\mathrm{~Pa}]$} \\
$D_{d}$ & 0.1086 & {$[\mathrm{~m}]$} \\
$d_{d}$ & 0.0076 & {$[\mathrm{~m}]$} \\
$D_{a}$ & 0.3153 & {$[\mathrm{~m}]$} \\
$d_{a}$ & 0.0122 & {$[\mathrm{~m}]$} \\
$\beta_{e, d}$ (eq.37) & $1.57 \mathrm{e} 9$ & {$[\mathrm{~Pa}]$} \\
$\beta_{e, a}$ (eq.37) & $1.45 \mathrm{e} 9$ & {$[\mathrm{~Pa}]$}
\end{tabular}

but the results of the PDE model still suggest that the used $\beta_{e}$ is slightly too big, illustrated by compression and expansion (flow change due to pressure) being smaller in the PDE model considering FSI than recorded flow data.

Figure 10 shows the PDE model compared to measured data for the third step in pressure, with and without FSI. For the third pressure step, the effect of considering FSI is smaller than in the case of the first two steps. The simulation with FSI show a slightly larger change in choke flow from changing choke pressure, as is the case for the first two steps as well as a slightly increased wave propagation time. The deviation between simulated flow and measured flow when the pressure is reduced might be caused by sensor inaccuracies. The dynamic performance of the Coriolis flow meter at transients as fast as in Figure 10, is uncertain.

\section{Conclusions}

The response of the commonly used simplified model by (Kaasa et al., 2012) and a distributed PDE based model has been compared to data from cased hole commissioning from an MPD system on an offshore well. It is shown that when changes are slow, the simplified and PDE based models show very similar response, matching quite closely that of the measured data. When the transient
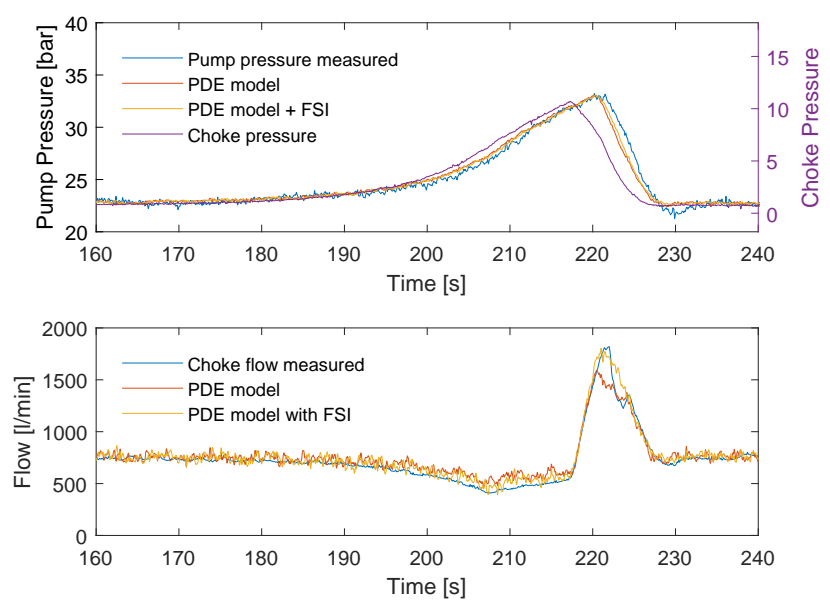

Figure 8. First step in choke position, comparison of original PDE-simulation and PDE-simulation considering fluid structure interactions. Top right axis: Measured choke pressure (boundary condition). Bottom: Simulated vs measured choke flow.
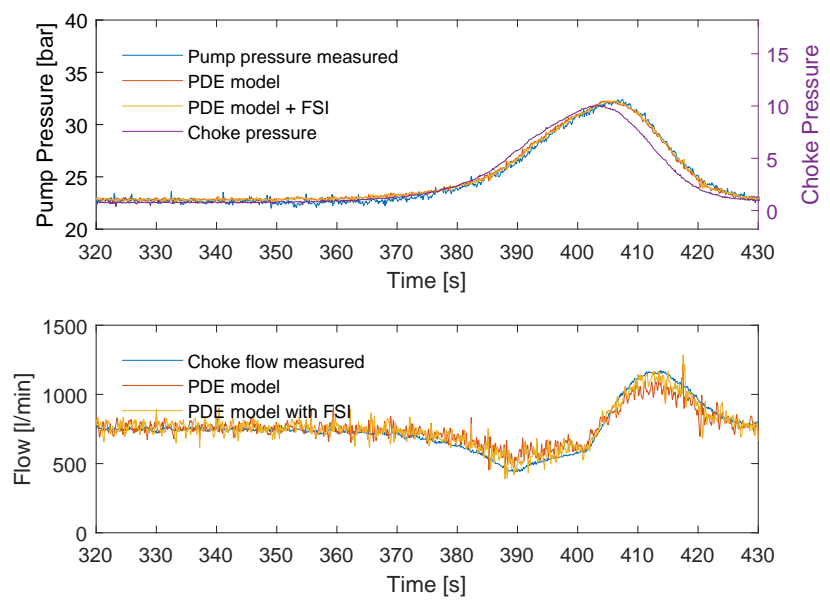

Figure 9. Second step in choke position. Slow closing and opening of choke, comparison of original PDE simulation and PDE simulation considering fluid structure interactions. Top right axis: Measured choke pressure (boundary condition). Bottom: Simulated vs measured choke flow.

changes are more rapid, a discrepancy between the simplified model and PDE model and measured data is seen. It is found that the PDE model under-predicts the effect of choke pressure on choke flow if only fluid properties are considered. When considering simple fluid structure interactions, the PDE model more closely fits the measured data. A discrepancy between the wave propagation time in the PDE model and measured data is observed. By manually "fudging" the system bulk modulus $\beta_{e}$, it is still not possible to make the PDE model fit with both choke flow and pump pressure (wave propagation time). To make the PDE model more closely fit the measured data, the well length or well volume and bulk modulus has to be changed. Well length and volume are considered quite well known, such that this result is indicative of something more fundamental missing from the PDE based model. The PDE model disregards 2-D effects on 

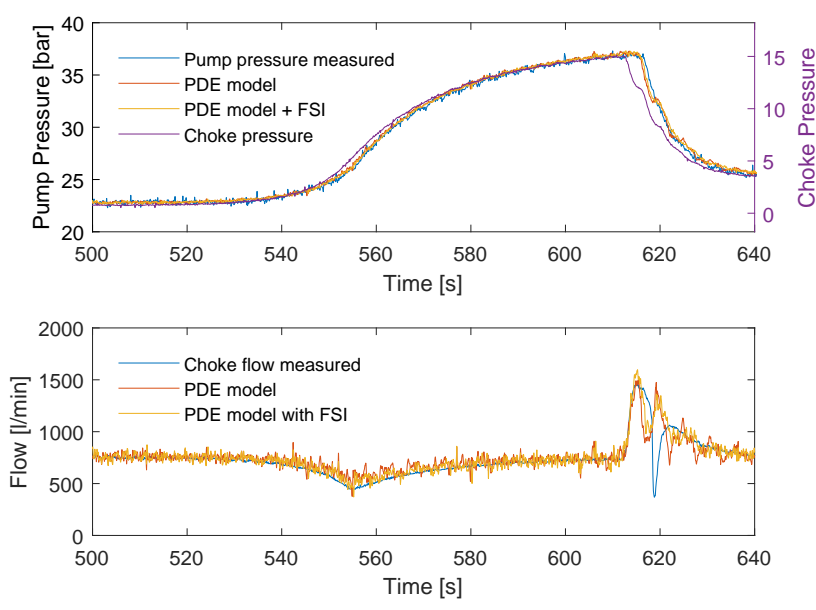

Figure 10. Third step in choke position. Very rapid opening of choke, comparison of original PDE simulation and PDE simulation considering fluid structure interactions. Top right axis: Measured choke pressure (boundary condition). Bottom: Simulated vs measured choke flow.

wave propagation, something that can increase the wave communication time. Further study of the discrepancy between the wave communication time in the 1D PDE model and recorded data, something that has been found in data from multiple wells, will require further work.

\section{Acknowledgements}

We thank Liobomyr Vytvysky on useful discussions on FSI in 1-D in compressible PDE models. This research has been partially funded by the the Norwegian Research Council in the Industrial PhD project "Modeling for automatic control and estimation of influx and loss in drilling operations" Project no 241586.

\section{References}

Ulf Jakob F Aarsnes, Ole Morten Aamo, and Alexey Pavlov. Quantifying error introduced by finite order discretization of a hydraulic well model. In Control Conference (AUCC), 2012 2nd Australian, pages 54-59, Aarsnes2012, 2012. IEEE. ISBN 1-922107-63-8.

Ulf Jakob Flø Aarsnes, Martin Standal Gleditsch, Ole Morten Aamo, and Alexey Pavlov. Modeling and Avoidance of Heave-Induced Resonances in Offshore Drilling. SPE Drilling \& Completion, 29(04):454-464, December 2014. doi:10.2118/173178-PA.

Henrik Anfinsen and Ole Morten Aamo. Adaptive OutputFeedback Stabilization of 2 x 2 Linear Hyperbolic PDEs with Actuator and Sensor Delay. In 2018 26th Mediterranean Conference on Control and Automation (MED), pages 1-6, Zadar, June 2018. IEEE. ISBN 978-1-5386-7890-9. doi:10.1109/MED.2018.8442627.

José M Carcione and Flavio Poletto. Sound velocity of drilling mud saturated with reservoir gas. Geophysics, 65(2): 646-651, 2000. ISSN 0016-8033.
Joel Carlsson. Water Hammer Phenomenon Analysis using the Method of Characteristics and Direct Measurements using a "stripped" Electromagnetic Flow Meter. Master Thesis, 2016.

Florent Di Meglio and Ulf Jakob Flø Aarsnes. A distributed parameter systems view of control problems in drilling. IFACPapersOnLine, 48(6):272-278, 2015.

Espen Hauge, Ole Morten Aamo, and John-Morten Godhavn. Model-based estimation and control of in/out-flux during drilling. In American Control Conference (ACC), 2012, pages 4909-4914, Hauge2012mbe, 2012. IEEE. ISBN 1-45771095-1.

Glenn-Ole Kaasa, Øyvind N Stamnes, Ole Morten Aamo, and Lars S Imsland. Simplified hydraulics model used for intelligent estimation of downhole pressure for a managed-pressure-drilling control system. SPE Drilling \& Completion, 27(01):127-138, 2012. ISSN 1064-6671. doi:10.2118/143097-PA.

Erika Palacios G and Carlos Da Silva. A finite volume study for pressure waves propagation in a straight section of pipeline with caviation. The International Journal of Multiphysics, 7(4):259-270, December 2013. ISSN 1750-9548. doi:10.1260/1750-9548.7.4.259.

Jon Åge Stakvik, Christian Berg, Glenn-Ole Kaasa, Ole Morten Aamo, and Urs Lehner. Adaptive Model Based Choke Control System for MPD Operations. In SPE-179714-MS, SPE, April 2016. Society of Petroleum Engineers. ISBN 978-161399-446-7. doi:10.2118/179714-MS.

Jon Åge Stakvik, Christian Berg, Glenn-Ole Kaasa, Robert Graham, and Antonio Torrealba. Model-Based Control in Managed Pressure Drilling. In SPE/IADC Drilling Conference and Exhibition. Society of Petroleum Engineers, 2017. ISBN 1-61399-501-6.

Øyvind Nistad Stamnes, Jing Zhou, Glenn-Ole Kaasa, and Ole Morten Aamo. Adaptive observer design for the bottomhole pressure of a managed pressure drilling system. In $D e$ cision and Control, 2008. CDC 2008. 47th IEEE Conference On, pages 2961-2966. IEEE, 2008. ISBN 1-4244-3123-9. doi:10.1109/CDC.2008.4738845.

Prabhata Swamee and Akalank K. Jain. Explicit equations for pipe-flow problems. ASCE J Hydraul Div, 102:657-664, May 1976.

Henk Versteeg and W Malalasekera. An Introduction to Computational Fluid Dynamics : The Finite Volume Method / H. K. Versteeg and W. Malalasekera. April 2019.

Liubomyr Vytvytsky and Bernt Lie. Comparison of elastic vs. inelastic penstock model using OpenModelica. In The 58th Conference on Simulation and Modelling (SIMS 58) Reykjavik, Iceland, September 25th - 27th, 2017, pages 20-28, September 2017. doi:10.3384/ecp1713820.

Jing Zhou, Ole Morten Aamo, and Glenn-Ole Kaasa. Switched control for pressure regulation and kick attenuation in a managed pressure drilling system. Control Systems Technology, IEEE Transactions on, 19(2):337-350, 2011. ISSN 10636536. 